\title{
ESO distant cluster survey: spectroscopy $\dagger$
}

\author{
C. Halliday ${ }^{1}$, B. Milvang-Jensen ${ }^{2}$, S. Poirier ${ }^{3}$, B. M. Poggianti ${ }^{1}$, \\ P. Jablonka ${ }^{3}$, A. Aragón-Salamanca ${ }^{4}$, R. Pelló ${ }^{5}$, R. P. Saglia ${ }^{2}$, \\ G. De Lucia ${ }^{6}$, L. Simard ${ }^{7}$, D. I. Clowe ${ }^{8}$, G. Rudnick ${ }^{6}$ \\ and S. D. M. White ${ }^{6}$ \\ ${ }^{1}$ Osservatorio Astronomico, vicolo dell'Osservatorio 5, 35122 Padova \\ ${ }^{2}$ Max-Planck Institut für extraterrestrische Physik, Giessenbachstrasse, D-85748 Garching \\ ${ }^{3}$ GEPI, CNRS-UMR8111, Observatoire de Paris, F-92195 Meudon Cedex, France \\ ${ }^{4}$ School of Physics and Astronomy, University of Nottingham, NG7 2RD, UK \\ ${ }^{5}$ Laboratoire d'Astrophysique, UMR 5572, Observatoire Midi-Pyrenees, 31400 Toulouse \\ ${ }^{6}$ Max-Planck-Institut für Astrophysik, Karl-Schwarzschild-Str. 1, D-85741 Garching \\ ${ }^{7}$ Herzberg Institute of Astrophysics, N. R. C. of Canada, Victoria, BC V9E 2E7, Canada \\ ${ }^{8}$ Steward Observatory, University of Arizona, 933 North Cherry Avenue, Tucson, AZ 85721
}

\begin{abstract}
We present first results for spectroscopic observations of galaxies in 4 clusters at $z=0.7-0.8$ and one cluster at $z=0.5$ observed by the ESO Distant Cluster Survey (EDisCS). Our spectroscopic catalogues contain 236 cluster members of our 5 clusters, and the number of members per cluster ranges from 30 to 67 . Our cluster velocity dispersions are between $\sim 400$ and over $1000 \mathrm{~km} \mathrm{~s}^{-1}$. Galaxy redshift distributions are found to be non-Gaussian and we find evidence for significant substructure in two clusters, one at $z \sim 0.79$ and another at $z \sim 0.54$; both clusters have velocity dispersions exceeding $1000 \mathrm{~km} \mathrm{~s}^{-1}$. These systems have clearly not yet virialised at these epochs in qualitative agreement with CDM scenarios and their cluster velocity dispersions should not be used in the measurement of cluster mass. Our clusters have a wide range of different cluster velocity dispersions, richnesses and substructuring, and our spectroscopic data set is allowing a comprehensive insight into cluster galaxy evolution as a function of redshift and environment.
\end{abstract}

\section{Introduction}

We present first spectroscopic results for the ESO Distant Cluster Survey (EDisCS). EDisCS (PI: White), is a photometric and spectroscopic survey of galaxies in 20 clusters at redshifts 0.4 to 0.8 selected from the Las Campanas Distant Cluster Survey (LCDCS) (Gonzalez et al. 2001). A first test of cluster reality for our EDisCS clusters was completed using imaging in two passbands (Gonzalez et al. 2002). The EDisCS project is investigating galaxy morphology, structure, masses, stellar populations and star formation histories as a function of both redshift and cluster environment, and study cluster mass and structure. Our programme involves deep optical photometry with the VLT (14 nights) (White et al. in preparation), near-IR photometry with the NTT (20 nights) (Aragón-Salamanca et al. in preparation), and multi-slit spectroscopy using the FORS2 spectrograph at the VLT (20 nights) (Halliday et al. A\&A accepted; Milvang-Jensen et al. in preparation). This ground-based data set has been complemented by 80 orbits of HST ACS imaging (PI: Dalcanton) for 10 EDisCS clusters (Desai et al. in preparation).

$\dagger$ Based on observations collected at the European Southern Observatory, Chile (166.A-0162). 
Cluster name No. of members $z_{\text {cluster }} \sigma_{\text {cluster }} \pm \delta \sigma_{\text {cluster }} \quad \mathrm{P}_{D S}$

\begin{tabular}{lcccc}
$(1)$ & $(2)$ & $(3)$ & $(4)$ & $(5)$ \\
\hline $\mathrm{Cl} 1040$ & 30 & 0.7043 & $418_{-46}^{+55}$ & 0.08 \\
$\mathrm{Cl} 1054-11$ & 49 & 0.6972 & $589_{-70}^{+78}$ & 0.64 \\
$\mathrm{Cl} 1054-12$ & 36 & $0.7498_{-113}$ & $504_{-65}^{+113}$ & 0.14 \\
$\mathrm{Cl} 1216$ & 67 & 0.7943 & $1018_{-77}^{+73}$ & 0.05 \\
$\mathrm{Cl} 1232$ & 54 & 0.5414 & $1080_{-89}^{+119}$ & 0.01 \\
\hline
\end{tabular}

Table 1. Cluster members and velocity dispersions are presented for each EDisCS cluster indicated in column 1; column 2 - number of cluster members; column 3 - cluster redshift; column 4 - cluster velocity dispersions $\left(\sigma_{\text {cluster }}\right)$ and errors in $\mathrm{km} \mathrm{s}^{-1}$; and column 5 - measure of probability of cluster substructuring from a Dressler-Shectman analysis.
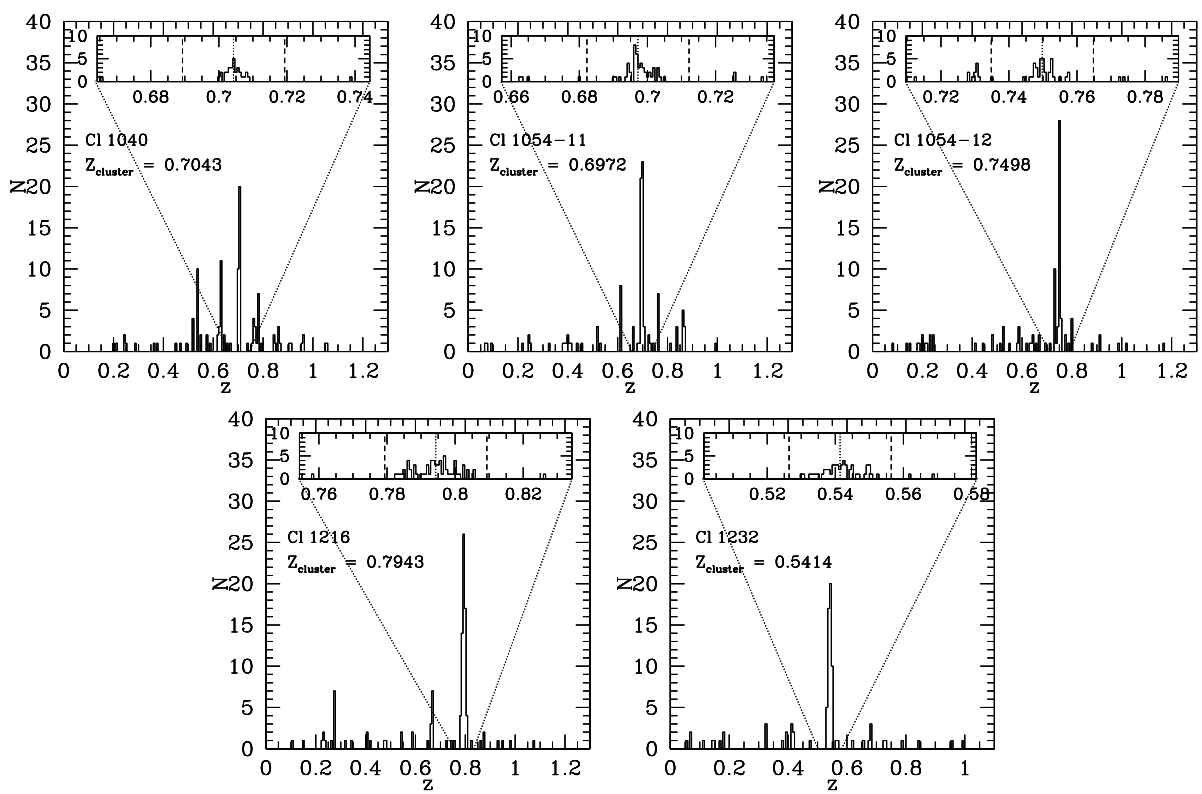

Figure 1. Redshift histograms for all 5 EDisCS clusters. Inset panel of each plot shows zoomed histogram for the redshift interval $z_{\text {cluster }}-0.04 \leqslant z_{\text {cluster }} \leqslant z_{\text {cluster }}+0.04$, where the cluster redshift $z_{\text {cluster }}$ is indicated by a dotted line. Dashed lines indicate the interval $z_{\text {cluster }}-0.015 \leqslant z_{\text {cluster }} \leqslant z_{\text {cluster }}+0.015$. Redshift bins are $0.00625\left(1874 \mathrm{~km} \mathrm{~s}^{-1}\right)$ for main plot and in inset plot $0.000625\left(187 \mathrm{~km} \mathrm{~s}^{-1}\right)$ (observed frame).

\section{Spectroscopy of EDisCS: first results}

Deep spectroscopic exposures have been obtained to date for 9 of our high- $z$ clusters and 4 of our low- $z$ clusters. Galaxies were selected from our I-band photometric catalogues to have I magnitude $<22$ for our low- $z$ clusters, and I $<23$ for our high$z$ clusters. Probabilities of cluster membership and star and galaxy classification were generated using our photometric redshift codes (Pelló, Rudnick et al. in preparation). Our spectroscopy has defined cluster membership and we are constraining the ages and metallicities of cluster absorption-line galaxies using evolutionary population synthesis models and studying early- and late-type galaxy evolution using emission line equivalent widths, the Fundamental Plane and Tully-Fisher scaling relations and interpretation with galaxy morphologies. We present first results for the VLT spectroscopy data of 5 clusters (4 high- $z$ and 1 low- $z$ ) from Halliday et al. Our redshift histograms in Figure 1 show that 

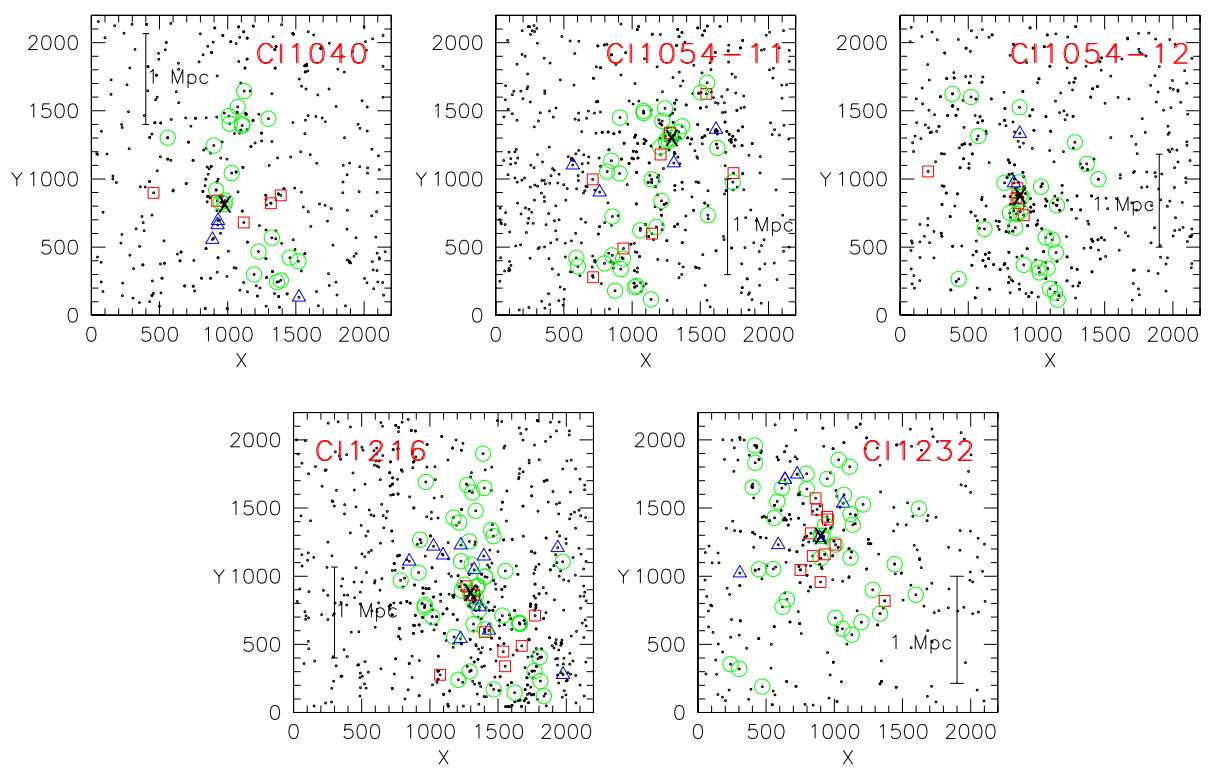

Figure 2. Plots of galaxy positions. Axis scales are in units of CCD pixels ( $0.2 \mathrm{arcsec} / \mathrm{pixel})$. The cluster name is indicated at the top of each plot. Open symbols indicate the positions of cluster members: green open circles show galaxies within \pm one $z_{\sigma}$ from the cluster redshift $z_{\text {cluster }}$, red open squares indicate galaxies whose redshift is more than one $z_{\sigma}$ higher than $\mathrm{z}_{\text {cluster }}$, and blue open triangles represent galaxies whose redshift is lower than $\mathrm{z}_{\text {cluster }}$ by more than one $z_{\sigma}$, where $z_{\sigma}$ is the redshift interval corresponding to the cluster velocity dispersion in the observed frame. Dots mark positions of galaxies selected from our I-band photometric catalogues as possible cluster members. The position of the brightest cluster galaxy (BCG) is given by a black cross symbol. A distance of $1 \mathrm{Mpc}$, calculated for a $\Lambda$ CDM cosmology with $\Omega=0.3, \Lambda=0.7$ and $h$ $=0.7$, is provided in each plot.

the cluster velocity distributions of the 5 EDisCS clusters are non-Gaussian. The XY positions of cluster members are given in Figure 2. For two clusters (Cl1232 and Cl1216), substructure is detected with more than $95 \%$ probability by a Dressler-Shectman (DS) analysis (Dressler \& Shectman 1988) (see Column 5 of Table 1). In Cl1232 the red-shifted galaxies are mainly in projection towards the cluster centre. In Cl1216 blue-shifted galaxies are offset North-East of the cluster centre. For two clusters no significant substructure is detected (Cl1040 and Cl1054-12). No evidence for substructure is found for Cl105411. In conclusion, the clusters of the highest cluster velocity dispersion exceeding 1000 $\mathrm{km} \mathrm{s}^{-1}$ (Cl1216 and Cl1232) display the most significant substructuring and are probably still undergoing collapse in qualitative agreement with CDM hierarchical scenarios. Their cluster velocity dispersions should not be used in the measurement of cluster mass.

\section{References}

Dressler, A. \& Shectman, S. A. 1988 A. J. 95, 985-995.

Gonzalez, A. H., Zaritsky, D., Dalcanton, J. J. \& Nelson, A. 2001 Ap. J. S. 137, 117-138.

Gonzalez, A. H., Zaritsky, D., Simard L., Clowe D. \& White, S. D. M. 2002 Ap. J. 579, 577-586. 